\title{
Redesigning safety regulation in the NHS
}

Charles Vincent and colleagues argue that patients will be better protected by a well designed, simpler, integrated regulatory system

\author{
Charles Vincent professor of psychology ${ }^{1}$, Eirini Oikonomou research fellow ${ }^{1}$, Jane Carthey human \\ factors and patient safety specialist ${ }^{2}$, Carl Macrae professor of organisational behaviour and \\ psychology $^{3}$
}

${ }^{1}$ Department of Experimental Psychology, 49 Walton Street, New Radcliffe House, University of Oxford, Oxford OX2 6AE, UK; ${ }^{2} J a n e$ Carthey Consulting, London, UK; ${ }^{3}$ Nottingham University Business School, Jubilee Campus, Wollaton Road, Nottingham NG8 1BB, University of Nottingham, UK

Healthcare relies on a variety of regulatory activities to manage risks to the public and to drive improvement. But the regulation of patient safety in healthcare, and in the NHS in particular, is "bewildering in its complexity and prone to both overlaps of remit and gaps between different agencies."1 Regulatory activities touch every single aspect of care delivery and place considerable demands on professionals and organisations alike, in the form of inspections, certification, accreditation, revalidation, and compliance reporting.

We argue that the safety regulatory system, as seen from the perspective of provider organisations, is much larger and more complex than usually supposed. Individual regulators might achieve valuable impact, but the system as a whole is unnecessarily burdensome, produces multiple unintended consequences, and, most importantly, fragments and dilutes regulatory impact. We discuss the nature of these problems and set out a series of practical proposals for tackling these critical challenges.

\section{What is regulation?}

Regulation can be defined as the "processes that aim to shape, motivate, monitor, and modify the practices and technologies within organisations so as to achieve some desired state of affairs." ${ }^{2}$ Regulatory activities take many forms, not all of them formal or backed by coercive or legal force. ${ }^{3}$ The work of regulating patient safety reaches from the "ivory towers" of policy making and standard setting to the "adobe huts" of local participation and frontline decision making. ${ }^{4}$

Regulation is achieved in multiple ways and by many different organisations. Statutory regulators, such as the Care Quality Commission and the General Medical Council, conduct inspections, monitor standards, and carry out a range of other activities. Many other organisations exert a regulatory effect on NHS organisations in the sense of inspecting, monitoring, influencing performance, and other activities (box 1, box 2).
Royal colleges, for example, set standards, review training, and influence organisations and individuals through the encouragement and enforcement of professional norms. They also set standards and have the power to impose sanctions, such as withdrawal of approval of training. 


\section{Box 1: Regulatory activities and definitions ${ }^{5}$}

Registration

Registration of healthcare professionals to ensure compliance with legal requirements

\section{Accreditation}

Accreditation, licensing, or revalidation to maintain and assure professional skills

\section{Policy provider}

Setting of formal rules and guidelines

Monitoring of services and professionals

Systematic collection of information to assess and maintain standards of care Investigation

Formal examination of an incident

Inspection

Formal examination or visit to assess standards of care

Imposition of sanctions

Penalties or fines for disobeying a law or rule

Quality improvement

Performance analysis and systematic attempt to improve it

Analysis and sharing of data

Collection of data, analysis, and potentially sharing with other regulatory organisations

\section{Advice and support for the public}

Publishing performance evaluations or other information on standards

\section{Advice and support for healthcare providers or other regulators}

Providing professional or legal advice to professionals and organisations

\section{Representation}

Representing professionals in the maintenance of standards

Professional development

Programmes of education and training to equip professionals with knowledge, skills, and competences

\section{Research}

Systematic investigation of events and information relevant to maintaining standards

\section{Box 2: Organisations with regulatory impact}

Defined as those who do all of the following ${ }^{5}$ :

- Consider the improvement of patient safety a part of their organisational responsibilities

- Undertake some form or monitoring of safety related standards or performance

- Attempt to influence the safety performance of NHS provider organisations

- Derive some form of legitimacy or external authority for their work on safety
}

\section{Regulation of patient safety in the NHS}

In 2002, in a paper discussing the rise of regulation in the NHS, Kieran Walshe argued that "current regulators vary widely in their statutory authority, powers, scope of action, and approach. The resulting mosaic of regulatory arrangements is highly fragmented, and some roles are duplicated." Since then, the complexity of the system has increased considerably, and several reports have argued for a less complex, more patient centred approach. $^{7-9}$ None of these reports, however, have described the totality of the regulatory system, making it difficult to make concrete plans for reform. We recently identified 126 organisations with regulatory influence that might interact with each English NHS trust (see supplementary figure).

Arrangements differ for Wales, Northern Ireland, and Scotland, but these systems face some similar problems.

In brief, NHS trusts are firstly responsible to the three national bodies that oversee the system and fund, lead, and support healthcare in England: the Department of Health and Social Care, NHS England, and Public Health England. In addition, they work directly with several clinical commissioning groups that carry out regulatory functions, such as requiring and monitoring reports on serious incidents.

In total, 17 statutory regulators with a specific remit for patient safety oversee healthcare institutions, professionals, and clinical procedures. There are also two agencies overseeing the management of medical products and a host of other regulators that operate across all industries to manage hazardous substances and practices such as radiotherapy.

We identified over 100 other organisations that might exert a regulatory influence on the NHS through their actions and activities. ${ }^{5}$ This group includes national agencies (such as the National Institute for Health and Care Excellence and the Healthcare Safety Investigation Branch), professional bodies (such as the Royal College of Physicians), and organisations that seek patient views, which are used to improve services (such as Healthwatch England). These organisations might not see themselves as regulators, but nonetheless they carry out some regulatory activities: many of them set standards, ask for data, and carry out inspections, and some are able to impose sanctions. They, therefore, have a major regulatory impact on provider organisations.

\section{Unintended consequences of the current system}

Individual regulatory organisations can undoubtedly have positive effects, but the system has many unintended consequences for both providers and regulatory organisations.

\section{Duplication of effort}

Despite efforts to improve information sharing, different bodies continue to request the same safety information in different formats. ${ }^{10}$ Several different bodies collect information on safeguarding, consent, infection control, staff training, and medicines management, for example, albeit for slightly different purposes. The net effect is that trusts have to repackage information collated for one regulator to meet the specific requirements of another. ${ }^{8}$ The Care Quality Commission is developing a more streamlined information system for sourcing data, ${ }^{11}$ but there is a limit to what it can achieve alone in such a complex landscape.

\section{Variability in approach}

The Professional Standards Authority has pointed out that all nine of the professional regulatory bodies it oversees have a common set of functions, yet they have pronounced differences in legislation, standards, and approach. ${ }^{12}$ So different NHS professionals are regulated differently, with almost no integration between the regulation of individuals and the organisations they work in.

\section{Fragmented approach}

Regulatory organisations manage numerous jurisdictional boundaries between each other. The Francis Inquiry found that the responsibilities and accountabilities of external agencies 
were not well defined, often resulting in "regulatory gaps" or failure to follow up warning signs. ${ }^{13}$ Criticism might be made of specific organisations, but the more fundamental problem is the plethora of agencies and the lack of clarity about who is responsible for oversight of which services. By contrast, aviation and other safety critical industries have a clearly defined regulatory structure, usually with a single dominant central regulator (such as the Civil Aviation Authority), so the lines of responsibility and accountability are much clearer.

\section{Culture of compliance rather than safety improvement}

Leadership attention is a scarce resource. If executives and clinical leaders are too busy meeting multiple (sometimes conflicting) regulatory standards, they have less chance of anticipating and resolving emerging patient safety risks in their organisations. ${ }^{14}$ NHS boards can become so focused on meeting external standards that a culture of compliance predominates, and "regulatory myopia" sets in. In these circumstances, safety and quality improvement is marginalised in favour of a display of compliance, which might degenerate into a denial of risks and safety challenges.

\section{Dilution of impact}

The impact of any intervention by one regulator, however important, is diluted because NHS trusts are also attempting to respond to many other agencies that are making similar but not identical requests. Almost inevitably, trusts will fall back on demonstrations of compliance rather than the more substantial long term improvements that both regulators and trusts would prefer.

\section{What practical steps can be taken?}

Regulating the safety of healthcare is complex, but the current regulatory system is unnecessarily so and needs urgent reform. This might seem to require yet another high level reorganisation with all the accompanying disruption. But we think that much could be achieved with a collaborative programme to systematically rethink, harmonise, and integrate the activities of the multiple bodies with regulatory influence. If reform or statutory changes are needed, they should follow, not precede, the work of synthesis and integration.

No single healthcare regulator can tackle these systemic problems alone, but trying to coordinate 126 organisations in a reform programme would lead to frustration and stasis. A small group of major regulatory organisations, however, could begin the process, model an approach, and seek some early gains (box 3). We suggest that the Care Quality Commission should take the lead in forming a small group of regulatory organisations that would each commit a small resource (one or two members of staff each initially) to begin the process of simplifying and harmonising the system.
Box 3: 10 practical approaches to improve regulatory efficiency and effectiveness

Develop a common language, a set of objectives, and an understanding of regulation across regulatory organisations

Harmonise the format and timing of data collection from trusts across regulatory organisations

Harmonise standards across regulators to abolish conflict and reduce replication

Identify and reduce duplication of regulatory activity in which multiple regulators oversee the same clinical processes

Zero tolerance of "regulatory repackaging requests," in which healthcare providers are asked to repackage the same information multiple times in different formats

Map the complete regulatory demands on each trust and assess the overall benefit, risks, and financial and opportunity costs

Collate evidence on the extent to which resources allocated to patient safety improvement work by trusts is diverted by regulatory inspections and demands

Compare the proportions of trust staff engaged in meeting regulatory demands with those of staff whose principal role is safety and quality improvement

Inspect, support, and encourage improvement activity rather than only monitoring compliance

Simplify the regulatory structure for patient safety ensuring there is a single agency identified as the lead regulator for health and social care.

\section{Integrate and harmonise regulatory structures and activities}

In the short term, small steps could be taken with fairly straightforward, time limited projects such as harmonising the format and timing of data collection, mapping duplication of effort between participating organisations, identifying non-productive regulatory activity, and identifying areas in which one regulator could draw on the findings of another without re-inspecting the same organisation. Even cursory inquiries to trusts show multiple examples of duplication of effort that could be resolved relatively easily with considerable savings in time and resources.

\section{Develop a shared framework of regulatory objectives, models, and language}

A common language and set of shared ideas and agreed objectives is essential when attempting to coordinate any sort of regulatory activity - and this has been achieved over time in many other industries. ${ }^{15}$ Health regulators have been urged to develop a shared "theory of regulation" that encompasses a common purpose, common objectives, and a shared understanding of the association between regulation and improvement. ${ }^{12}$

In practice, this would include the development of objectives, terminology, and practices as well as a shared understanding of how regulation best achieves its intended effects. The broad range of risks that healthcare regulation is seeking to manage - from misdiagnosis to inappropriate care and emotional harm - should be clearly articulated. The core group could lead initial work then seek wider consultation and engagement, which would allow further harmonisation across the system.

\section{Evaluate the activities, costs, and benefits of regulation across the entire system}

The regulatory system has changed over time, with a gradual accretion of new functions, bodies, and requirements. A systematic and detailed mapping of the activities, impact, and cost of regulatory activities is required. This should include a full recording and costing of all the time spent by trusts responding to regulatory requests of all kinds from all relevant 
organisations. This is ambitious and complex but essential if improvements are to be well targeted and sustained.

\section{Long term coordination and integration}

Healthcare is complex and probably cannot be overseen by a single regulator. In the longer term, however, it should be possible to greatly reduce the number of organisations and identify a single body to coordinate and integrate the overall regulatory system. Such a body, which could be a coalition of several of the major regulators, would seek to integrate regulatory activity along the entire patient journey, considering care within organisations, across patient transitions, and in community settings.

\section{Conclusions}

Sensible and proportionate regulation should be an essential foundation of safe, high quality patient care but is not remotely achievable in the present system. The regulatory system of the NHS is not fully understood even by professional regulators and government; it is almost impossible for the general public to navigate or even understand it.

Complex systems need to be carefully designed to ensure that they serve their intended functions. A broad, participative, and practical programme of system analysis and design is needed, encompassing everything from the overarching principles to the practicalities of data collection, communication, and influence. This is a necessary foundation for the longer term objective of a more responsive, improvement focused relationship between regulators and providers of services.

This is ambitious but entirely feasible. Much can be achieved with no major reorganisation or statutory reform. The benefits of reforming NHS regulation are considerable for regulators, providers, and, most importantly, patients and families, who will be much better protected in a simpler, sharper regulatory system.

\section{Key messages}

- The current regulatory system of the NHS is burdensome for both providers and regulators, has multiple unintended consequences, and fragments and dilutes regulatory impact

- The system needs to be redesigned to be efficient and adaptable for both the regulators and the organisations they oversee

- The Care Quality Commission should coordinate a small group of major regulatory organisations to reform the system

- Primary initial tasks are to achieve a common understanding of objectives and methods and to harmonise and integrate data collection and other regulatory activities
We thank the Health Foundation for funding the original research. We thank Gwyn Bevan, Ted Baker, and Paul Simpson for comments on an earlier draft of this paper. The views expressed are those of the authors.

Contributors and sources: $\mathrm{CV}$ is professor of psychology at the University of Oxford and director of Oxford Healthcare Improvement. His research is concerned with understanding how healthcare can become safer. $\mathrm{CM}$ is professor of organisational behaviour and psychology at the University of Nottingham. His research explores how organisations achieve high levels of safety, reliability, and resilience, and his professional career spans research, policy, and regulation in healthcare and other industries. JC is a human factors consultant with 17 years' experience working at both a national and local level in the NHS, applying human factors science to improve safety and quality. EO is a research fellow at the department of psychology at the University of Oxford and conducted a mapping study of regulation in the NHS. We sourced information from our companion paper published in BMJ Open and from a programme of research funded by the Health Foundation. The recommendations are based on the mapping exercise itself; case studies of trusts; a seminar of stakeholders held during the research process; the wider regulation literature, including other industries; and our own observations and experience in the NHS. CV is the guarantor of this article.

Patient involvement: Not applicable for this project.

Competing interests: We have read and understood BMJ policy on competing interests and have no relevant interests to declare.

1 Berwick D. A promise to learn-a commitment to act: improving the safety of patients in England. London; 2013.

2 Hutter BM, ed. Compliance: regulation and environment. Clarendon Press, 1997.

3 Yeung K, Dixon-Woods M. Design-based regulation and patient safety: a regulatory studies perspective. Soc Sci Med 2010;71:502-9.

10.1016/j.socscimed.2010.04.017 20538395

4 MacRae C. Regulating resilience? Regulatory work in high-risk arenas. In: Hutter BM, ed. Anticipating risks and organizing risk regulation. Cambridge University Press, 2010: 139-60, 10.1017/CBO9780511761553.008

5 Oikonomou E, Carthey J, Macrae C, Vincent C. Patient safety regulation in the NHS: mapping the regulatory landscape of healthcare. BMJ Open 2019;9:e028663. 10.1136/bmjopen-2018-028663 31289082

6 Walshe K. The rise of regulation in the NHS. BMJ 2002;324:967-70. 10.1136/bmj.324.7343.967 11964345

7 NHS Providers. Maximising the value from the regulatory approach. 2015. https:// nhsproviders.org/resource-library/reports/maximising-the-value-from-the-regulatoryapproach

8 NHS Providers. The impact of regulation in a shifting environment. 2017. https:// nhsproviders.org/resource-library/reports/impact-of-regulation-in-a-shifting-environment

9 Dixon-Woods M, Pronovost PJ. Patient safety and the problem of many hands. BMJ Qual Saf 2016;25:485-8. 10.1136/bmjqs-2016-005232 26912578

10 Edwards N. Burdensome regulation of the NHS. BMJ 2016;353:i3414. 10.1136/bmj.i3414 27325610

11 Smithson R, Richardson E, Roberts J, et al. Impact of the Care Quality Commission on provider performance. Room for improvement? 2018. https://www.kingsfund.org.uk/sites/ default/files/2018-09/cqc-provider-performance-report-september2018.pdf

12 Professional Standards Authority. Rethinking regulation. 2015. www.professionalstandards. org.uk

13 Thorlby R, Smith J, Williams S, Dayan M. The Francis report: one year on. 2014. www. nuffieldtrust.org.uk/our-work/projects/francis-inquiry

14 Vincent C, Burnett S, Carthey J. Safety measurement and monitoring in healthcare: a framework to guide clinical teams and healthcare organisations in maintaining safety. BMJ Qual Saf 2014;23:670-7. 10.1136/bmjqs-2013-002757 24764136

15 Macrae C. Close calls: managing risk and resilience in airline flight safety. Springer, 201410.1057/9781137376121

Published by the BMJ Publishing Group Limited. For permission to use (where not already granted under a licence) please go to http://group.bmj.com/group/rights-licensing/ permissions 